Georg Altmann

Unternehmensführung und Innovationserfolg 
GABLER EDITION WISSENSCHAFT 
Georg Altmann

\section{Unternehmensführung und Innovationserfolg}

Eine empirische Untersuchung

im Maschinenbau

Deutscher Universitäts-Verlag 
Bibliografische Information Der Deutschen Bibliothek

Die Deutsche Bibliothek verzeichnet diese Publikation in der Deutschen Nationalbibliografie; detaillierte bibliografische Daten sind im Internet über <http://dnb.ddb.de> abrufbar.

Dissertation Universität München, 2002 u.d.T.: Altmann, Georg: Der innovative Unternehmer. Eine empirische Analyse

D19

\section{Auflage April 2003}

Alle Rechte vorbehalten

(C) Deutscher Universitäts-Verlag GmbH, Wiesbaden, 2003

Lektorat: Brigitte Siegel / Annegret Eckert

Der Deutsche Universitäts-Verlag ist ein Unternehmen der Fachverlagsgruppe BertelsmannSpringer.

www.duv.de

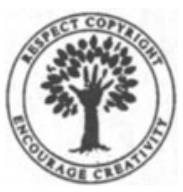

Das Werk einschließlich aller seiner Teile ist urheberrechtlich geschützt. Jede Verwertung außerhalb der engen Grenzen des Urheberrechtsgesetzes ist ohne Zustimmung des Verlags unzulässig und strafbar. Das gilt insbesondere für Vervielfältigungen, Übersetzungen, Mikroverfilmungen und die Einspeicherung und Verarbeitung in elektronischen Systemen.

Die Wiedergabe von Gebrauchsnamen, Handelsnamen, Warenbezeichnungen usw. in diesem Werk berechtigt auch ohne besondere Kennzeichnung nicht zu der Annahme, dass solche Namen im Sinne der Warenzeichen- und Markenschutz-Gesetzgebung als frei zu betrachten wären und daher von jedermann benutzt werden dürften.

Umschlaggestaltung: Regine Zimmer, Dipl.-Designerin, Frankfurt/Main

Gedruckt auf säurefreiem und chlorfrei gebleichtem Papier

ISBN-13:978-3-8244-7842-2

e-ISBN-13:978-3-322-81522-4

DOI: $10.1007 / 978-3-322-81522-4$ 


\section{Vorwort}

Innovationen sind die Grundvoraussetzung für den langfristigen Erfolg von Unternehmen. Dies gilt besonders für den Maschinenbau, einen der weltweit innovativsten Industriezweige. Es ist daher eine wichtige Aufgabe der anwendungsorientierten Wissenschaft zu erforschen, wie Innovationen erfolgreich geführt und umgesetzt werden können. Die vorliegende Arbeit nimmt sich dieser Aufgabe an und untersucht erstmals neben organisatorischen Faktoren auch Verhalten und Persönlichkeit der höchsten Führungskraft eines Unternehmens. Das umfassende methodische Instrumentarium, das bei der Gewinnung der empirischen Ergebnisse eingesetzt wurde, ist die Grundlage sowohl für den empirischen Nachweis der hohen Bedeutung des Unternehmers für Innovationen als auch für konkrete Verbesserungsvorschläge für Unternehmen, die an der Optimierung ihres Innovationsmanagements interessiert sind.

Diese Arbeit entstand während meiner Zeit als Assistent am Institut für Innovationsforschung, Technologiemanagement und Entrepreneurship der Ludwig-MaximiliansUniversität München. Sie ist das Ergebnis eines gemeinsamen Forschungs- und Beratungsprojektes des Instituts mit dem Zentrum für Europäische Wirtschaftsforschung (ZEW) und dem Verband Deutscher Maschinen- und Anlagenbau (VdMA). Im Juli 2002 wurde die Arbeit von der Fakultät für Betriebswirtschaftslehre der LudwigMaximilians-Universität München als Dissertation angenommen.

Mein besonderer Dank gilt Prof. Dr. Stephan Schrader, Prof. Dr. Dres. h.c. Eberhard Witte und Prof. Dietmar Harhoff, Ph.D. Ohne sie wäre diese Arbeit nicht möglich gewesen. Prof. Dr. Dres. h.c. Eberhard Witte und Prof. Dietmar Harhoff, Ph.D. haben die Arbeit nach dem frühen Tod von Prof. Dr. Stephan Schrader betreut und mich in jeder Hinsicht mit Rat und Förderung unterstützt. Dafür danke ich Ihnen herzlich.

Danken möchte ich auch den zahlreichen Unternehmern mittelständischer Maschinenbauunternehmen, die mit ihrer Gesprächsbereitschaft und Unterstützung ganz erheblich zu dieser Arbeit beigetragen haben. Auch den beteiligten Führungskräften und Mitarbeitern des Zentrums für Europäische Wirtschaftsforschung (ZEW) sowie des Verbandes Deutscher Maschinen- und Anlagenbau (VdMA) möchte ich für die gute Zusammenarbeit und die Unterstützung danken.

Zahlreiche Diskussionspartner haben zum Fortgang des Forschungsprozesses maßgeblich beigetragen. Besonders bedanken möchte ich mich bei Prof. Dr. Nikolaus Franke, Dr. Marc Gruber, Dr. Joachim Henkel, Dr. Claudia Küpper, Dr. Georg Licht und Dr. 
Christian Lüthje. Danken möchte ich nicht zuletzt Karin Hoisl, Marc Jokisch, Christian Rasim und Steffen Wiedemann, die mich durch ihre hervorragende Mitarbeit und ihren unermüdlichen Einsatz unterstützt haben. Mein Dank gilt abschließend auch all jenen hier nicht genannten Personen, die zu dieser Arbeit beigetragen haben.

Georg Altmann 


\section{Inhaltsverzeichnis}

Abbildungsverzeichnis........................................................................................................ XI

Tabellenverzeichnis.......................................................................................................... XIII

Abkürzungsverzeichnis ............................................................................................................... XV

1 Einleitung.............................................................................................................................1

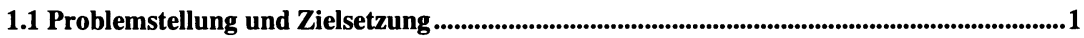

1.2 Vorgehensweise und Aufbau .........................................................................................................3

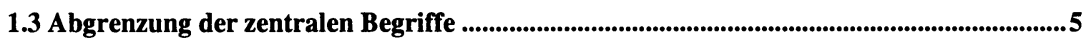

1.4 Auswahl und Besonderheiten des empirischen Feldes...........................................................11

2 Theoretische Grundlagen und empirische Bestandsaufnahme ............................15

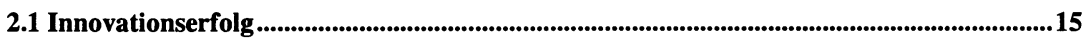

2.1.1 Der Innovationserfolg als mehrdimensionales Konstrukt ............................................................... 16

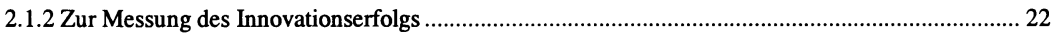

2.2 Person und Innovation: Der Unternehmer ...........................................................................................25

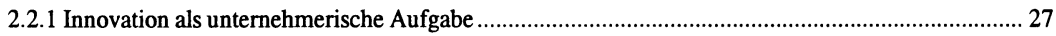

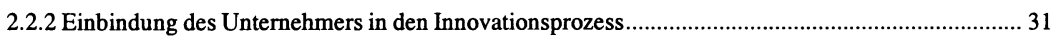

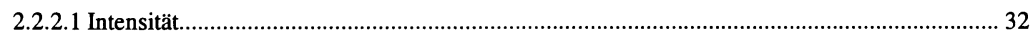

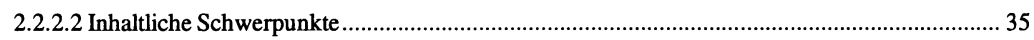

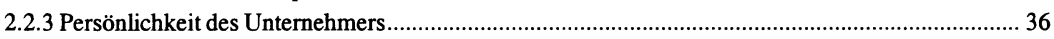

2.3 Organisation und Innovation: Innovationsmanagement.................................................41

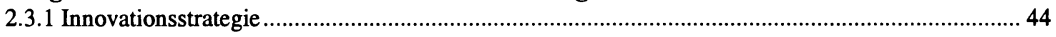

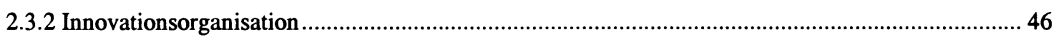

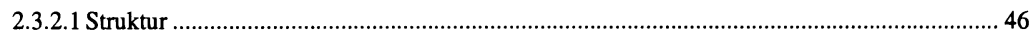

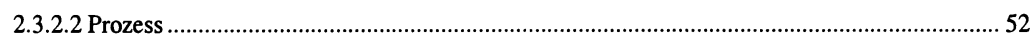

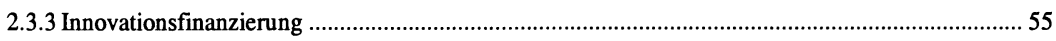

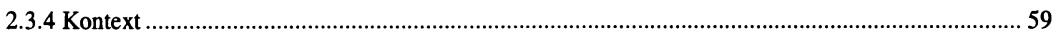

2.4 Zusammenfassung: Das Hypothesensystem im Überblick............................................61 
3 Methodik und Konzeption der empirischen Analyse ...................................................665

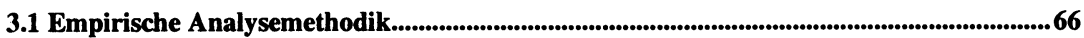

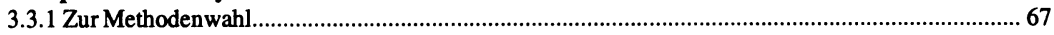

3.1.2 Varianten und Anwendungsvoraussetzungen der multiplen Regressionsanalyse.............................69

3.2 Pilotstudien...................................................................................................................................................72

3.2.1 Pilotstudie 1: Exploration durch Expertengespräche ……………................................................. 73

3.2.2 Pilotstudie 2: Skalenkonstruktion zur Messung der Unternehmerpersönlichkeit.............................. 75

3.2.3 Pilotstudie 3: Zur Messung des Innovationserfolgs .................................................................... 79

3.3 Haupterhebung .........................................................................................................................................8

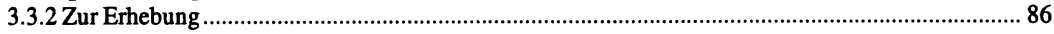

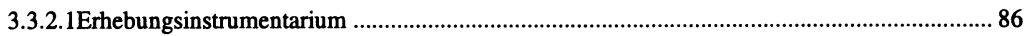

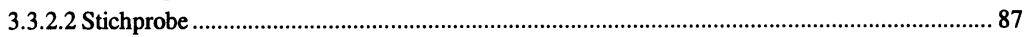

3.3.2.3 Vorbereitung und Durchführung der Erhebung.................................................................... 89

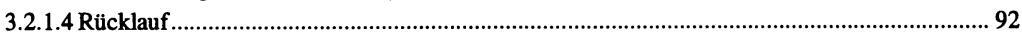

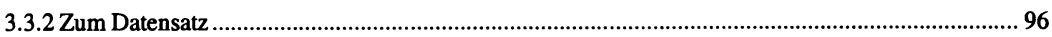

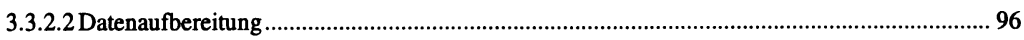

3.3.2.3 Non-Response-Analyse ............................................................................................... 99

3.4 Zusammenfassung ...................................................................................................................................104

4 Deskriptive und multivariate Partialanalysen ..............................................................105

4.1 Charakteristika der Unternehmen und Produktinnovationen ...............................................106

4.2 Innovationserfolg ..........................................................................................................................111

4.2.1 Deskriptive Erfolgsbeurteilung der analysierten Projekte............................................................. 112

4.2.2 Multivariate Überprüfung des mehrstufigen Innovationserfolgs-Modells ..................................... 114

4.2.3 Exkurs: Konsequenzen der unterschiedlichen Erfolgsbeurteilung durch Unternehmer und Nicht-

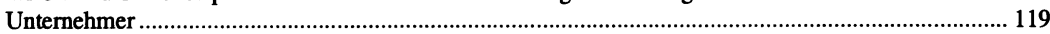

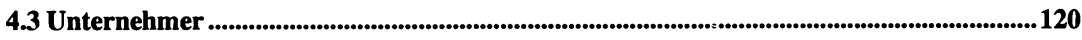

4.3.1 Einbindung des Unternehmers in den Innovationsprozess.......................................................... 120

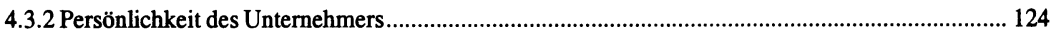

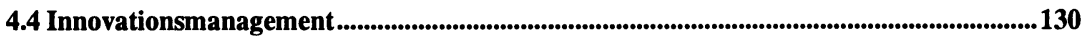

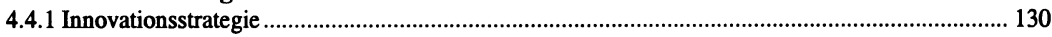

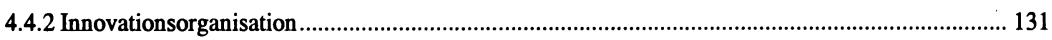

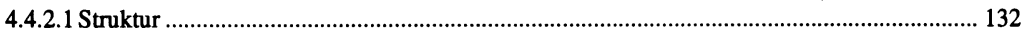

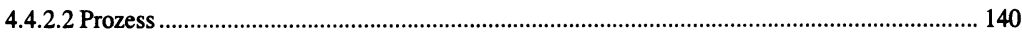

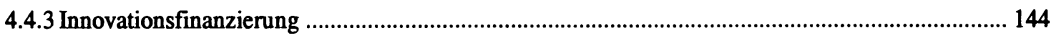

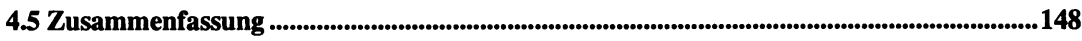


5 Multivariate Analysen: Determinanten des Innovationserfolgs..........................149

5.1 Multivariate Analysen und Optimierung der Modellstruktur ...............................................150

5.1.1 Das mehrstufige Erfolgsmodell als Ausgangspunkt...................................................................... 152

5.1.1.1 Die indirekten Erfolgsgrößen technischer Erfolg und Prozesserfolg ................................... 152

5.1.1.2 Zur Irrelevanz des Prozesserfolgs .................................................................................. 154

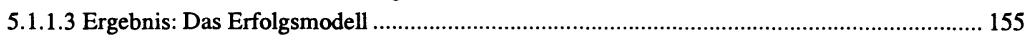

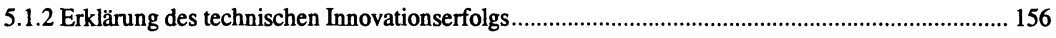

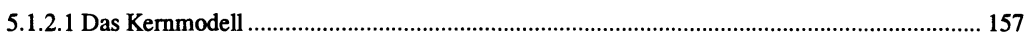

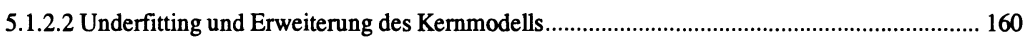

5.1.2.3 Overfitting und Reduktion des erweiterten Modells ......................................................... 163

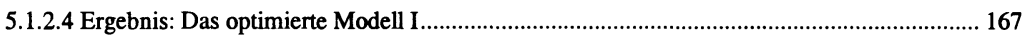

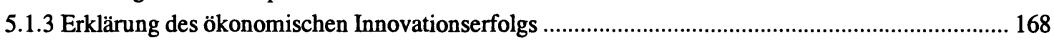

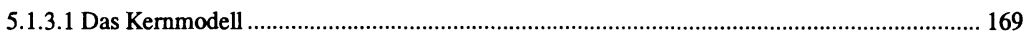

5.1.3.2 Underfitting und Erweiterung des Kernmodells.............................................................. 172

5.1.3.3 Overfitting und Reduktion des erweiterten Modells ........................................................... 174

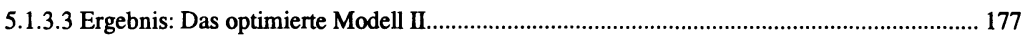

5.1.4 Das aggregierte Modell zur Erklärung des Innovationserfolgs .................................................... 178

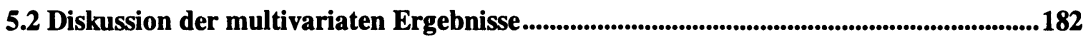

5.2.1 Vorbemerkung: Zur Bedeutung des Kontextes ......................................................................... 182

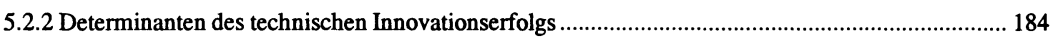

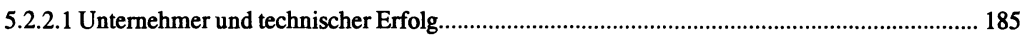

5.2.2.2 Innovationsmanagement und technischer Erfolg ............................................................ 187

5.2.2.3 Zusammenfassung: Determinanten des technischen Erfolgs ................................................... 188

5.2.3 Determinanten des ökonomischen Innovationserfolgs.......................................................... 190

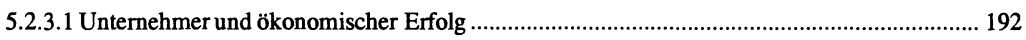

5.2.3.2 Innovationsmanagement und ökonomischer Erfolg ........................................................ 192

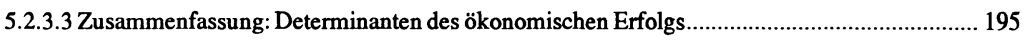

5.2.4 Zusammenfassung: Determinanten des Innovationserfolgs ........................................................... 197

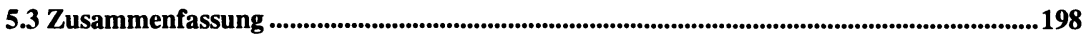

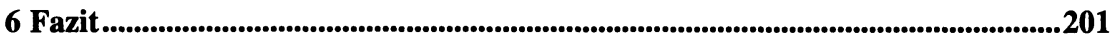

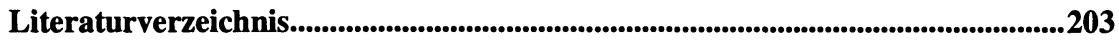

Anhang ...........................................................................................................................223 


\section{Abbildungsverzeichnis}

Abb. 1.1 Abgrenzung Innovations- und Technologiemanagement (Brockhoff) ...........7

Abb. 1.2 Unterschiede zwischen KMU und Großunternehmen (Pfohl) ......................14

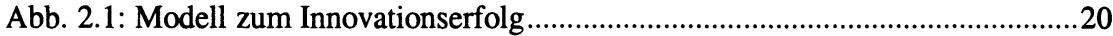

Abb. 2.2 Idealtypisches Modell zur Unternehmereinbindung (Gluck/Foster)..............34

Abb. 2.3: Übersicht über die nachfolgenden Abbildungen 2.4-2.6 ............................61

Abb. 2.4: Das Hypothesensystem zum Prozesserfolg................................................62

Abb. 2.5: Das Hypothesensystem zum technischen Innovationserfolg .......................63

Abb. 2.6: Das Hypothesensystem zum ökonomischen Innovationserfolg....................64

Abb. 3.1: Ablauf der empirischen Analysen .............................................................66

Abb. 3.2: Ablauf der Haupterhebung......................................................................91

Abb. 3.3: Zeitlicher Verlauf des Rücklaufs (Eingang der ausgefüllten Fragebögen)..92

Abb. 3.4: Grundgesamtheit, Auswahlgesamtheit und Rücklauf ..................................94

Abb. 3.5: Realisierter und ausgewerteter Rücklauf ..................................................95

Abb. 4.1: Kontext: Marktstellung des Unternehmens..............................................107

Abb. 4.2: Kontext: Hemmnisse im Innovationsmanagement ...................................108

Abb. 4.3: Strategische Bedeutung der Produktinnovation........................................110

Abb. 4.4: Fertigung der Produktinnovation .............................................................111

Abb. 4.5.: Der Unternehmer: Anteil der persönlichen Arbeitszeit ............................120

Abb. 4.6: Der Unternehmer: Engagement im Innovationsprozess ............................121

Abb. 4.8: Der Unternehmer: Inhaltliche Schwerpunkte der Unternehmereinbindung123 Abb. 4.9: Der Unternehmer: Erfahrung und Alter ....................................................127

Abb. 4.10: Der Unternehmer: Ausbildungsrichtung ...............................................128

Abb. 4.11: Der Unternehmer: Höchster Bildungsabschluss ......................................129

Abb. 4.12: Der Unternehmer: (Mit-)Inhaberschaft....................................................129

Abb. 4.13: Innovationsstrategie: Verfolgte strategische Ziele....................................130 
Abb. 4.14: Innovationsstrategie: Korrelation der strategischen Ziele ......................131

Abb. 4.15: Innovationsorganisation: Vertraglich geregelte Kooperation ..................132

Abb. 4.16: Innovationsorganisation: Informelle Kooperation mit Externen ..............133

Abb. 4.17: Innovationsorganisation: Anstöße zu der Produktinnovation...................134

Abb. 4.18: Innovationsorganisation: Projektübergreifende Ansprechpartner ............135

Abb. 4.19: Innovationsorganisation: Bereichsübergreifende Kooperation.................136

Abb. 4.20: Innovationsorganisation: Motivationsinstrumente....................................138

Abb. 4.22: Innovationsorganisation: Aufwand nach Phasen im Innovationsprozess 141

Abb. 4.23: Innovationsorganisation: Prozessorganisation...........................................142

Abb. 4.25: Innovationsfinanzierung: Durchschnittliches Finanzierungsportfolio.....144

Abb. 4.26: Innovationsfinanzierung: Finanzierungsarten I .......................................145

Abb. 4.27: Innovationsfinanzierung: Finanzierungsarten II .....................................147

Abb. 5.1: Zusammenfassung der untersuchten Erfolgsmodelle ................................151

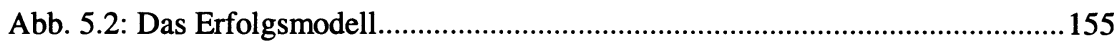

Abb. 5.3: Das Hypothesensystem: Determinanten des technischen Erfolgs...............189

Abb. 5.4: Das Hypothesensystem: Determinanten des ökonomischen Erfolgs .........196 


\section{Tabellenverzeichnis}

Tabelle 3.1: Reliabilitätskennzahlen der Persönlichkeitskonstrukte............................78

Tabelle 3.2: Korrelation des wahrgen. Gesamterfolgs mit Teilerfolgsindikatoren.......82

Tabelle 3.3: Erklärung des wahrgenommenen Gesamterfolgs (Modelle I + II) ..........84

Tabelle 3.4: Erklärung des wahrgenommenen Gesamterfolgs (Modelle III + IV) ......85

Tabelle 3.5: Probit-Modell zur Erklärung der Teilnahmewahrscheinlichkeit ............102

Tabelle 4.1: Die befragten Unternehmen: Deskriptive Statistiken ...........................106

Tabelle 4.2: Die einzelne Produktinnovation: Deskriptive Statistiken .......................109

Tabelle 4.3: Innovationsgrad der Produktinnovation: Deskriptive Statistiken ...........110

Tabelle 4.4: Erfolg der Produktinnovation: Deskriptive Statistiken............................112

Tabelle 4.5: Erfolg der Produktinnovation: Deskriptive Statistiken...........................114

Tabelle 4.6: Erklärung des wahrgenommenen Gesamterfolgs ...................................117

Tabelle 4.7: Skalenvalidierung (Persönlichkeitseigenschaften des Unternehmers) ..126

Tabelle 4.8: Innovationsorganisation: Projektorganisation ........................................137

Tabelle 5.1: Relevanz des technischen Erfolgs und des Prozesserfolgs....................153

Tabelle 5.2: Erklärung des technischen Erfolgs (Ausgangsmodelle) ........................ 159

Tabelle 5.3: Modellerweitungen zur Erklärung des technischen Erfolgs ...................161

Tabelle 5.4: Erklärung des technischen Erfolgs (Reduktion des Ausgangsmodells) 165

Tabelle 5.5: Erklärung des technischen Erfolgs (Das optimierte Modell I) .............. 168

Tabelle 5.6: Erklärung des ökonomischen Erfolgs (Ausgangsmodelle).....................171

Tabelle 5.7: Modellerweiterungen zur Erklärung des ökonomischen Erfolgs............174

Tabelle 5.8: Erklärung des ökonom. Erfolgs (Reduktion des Ausgangsmodells) .....175

Tabelle 5.9: Erklärung des ökonomischen Erfolgs (Das optimierte Modell II).........178

Tabelle 5.10: Erklärung des Innovationserfolgs (Das aggregierte Modell)............... 180

Tabelle 5.11: Determinanten des technischen Erfolgs............................................... 185

Tabelle 5.12: Determinanten des ökonomischen Erfolgs .........................................191

Tabelle 5.13: Determinanten des Innovationserfolgs ...............................................197 


\section{Abkürzungsverzeichnis}

BVW Betriebliches Vorschlagswesen

CATI Computer Aided Telephone Interview

CEO Chief Executive Officer

FuE Forschung und Entwicklung

GLS Generalized Least Square

KMO Kaiser-Meyer-Olkin-Kriterium

KMU Kleine und mittlere Unternehmen

MA Mitarbeiteranzahl (vollzeitäquivalent)

N Fallzahl

n.s. nicht signifikant auf dem 10\%-Niveau

OLS Ordinary Least Square

QFD Quality Function Deployment

r Korrelationskoeffizient

SF Standardfehler

VDMA Verband Deutscher Maschinen- und Anlagenbau

vgl. vergleiche

ZEW Zentrum für Europäische Wirtschaftsforschung 